\title{
Entrustable Professional Activities in Residency Programs - planning and scheduling issues
}

\author{
Jan Riezebos ${ }^{1}$, Durk Jouke van der Zee ${ }^{1}$, Jan Pruim ${ }^{2}$ \\ ${ }^{1}$ Department of Operations, University of Groningen, The Netherlands. \\ ${ }^{2}$ Chair Central Residency Committee , University Medical Center Groningen, University of \\ Groningen, The Netherlands.
}

\begin{abstract}
Residency programs allow residents, i.e., graduate physicians, to qualify for being a specialist in one of the medical disciplines. Their educational programme is strongly focusing on competencies and skills, but will incur a major change in the near future in order to introduce Entrustable Professional Activities (EPA's). EPA's focus on actual physician tasks and are assessed by supervisors in the clinic. The trust levels indicate whether trainees are ready for the next milestone, such as unsupervised practice, based on assessment of performance of those activities. EPA's have not only effect on the internships and the assessment process, but also impact the planning and scheduling of the educational programmes, internships, patient care services, and cooperation between regional hospitals. This study examines the effect on the planning and scheduling process and proposes a framework for planning and scheduling in order to facilitate the organization of this type of educational programs. The main improvements have to be found in an improved regional coordination between the hospitals, a more leading role for the educational programme directors, and more intelligent planning and scheduling.
\end{abstract}

Keywords: Educational organization, Planning and Scheduling; Learning for employment; Skill assessment. 


\section{Introduction}

Residency programs allow residents, i.e. graduate physicians, to qualify for being a specialist in one of the medical disciplines. A typical residency program lasts for around 4-6 years. It consists of internships and educational activities, such as workshops, trainings, et cetera. The internships allow residents to develop specific skills in practical settings under supervision of an attending physician. Residents learn by training on the job while at the same time contributing to the provision of care services. However, they are also involved in the provision of regular patient care services in the hospital during night shifts, weekends, et cetera, even though these activities do not serve a direct purpose for the educational program.

A major change in program setup and delivery is due to the adoption of a recent educational innovation in the field of competence-based learning and skills development: the use of Entrustable Professional Activities (EPA's) (Hauer et al., 2013; ten Cate, 2013) as the basis for assessment, rather than a time- or operation-based assessment. By describing the programme outcomes in terms of EPA's that should be performed at a predefined trust level, residency programs allow for more flexibility in terms of length of internship and reduce the inherent complexity of assessing competences. The change towards adopting EPA's has huge impact on many processes and consequences for professional standards the discipline has to adhere to, as has been described in literature (Englander \& Carraccio, 2014). However, the planning and scheduling problem that arises when implementing this educational innovation has not yet received attention in literature. As this innovation allows for different paces amongst the residents in the educational programme, planning and scheduling of their activities and regular care services cannot remain unchanged. This paper will focus on the required changes in planning and scheduling of residents' activities for the on-the-job training and patient care services they provide in hospitals. It aims to develop a framework for planning and scheduling that supports the change towards program delivery using EPA's.

\section{Planning and scheduling of residents' activities}

Planning and scheduling of residents' activities consider the time-based ordering of residents' activities within the program period. The planning and scheduling of residents' activities is considered to be complex and time consuming (Cohn, Root, Kymissis, Esses, \& Westmoreland, 2009; Ernst, Jiang, Krishnamoorthy, \& Sier, 2004; Güler, Idin, \& Yilmaz Güler, 2013). Outcomes of the planning and scheduling activities, i.e., programs, rosters, and schedules are often criticized by the various stakeholders, especially residents, educators, and the department management. Issues like the fragmentation of the educational schemes following from an integration of a residents' educational and remainder activities, the imbalance of supply and demand for educators, and the balancing of residents' and management interests in scheduling non-educational activities are frequently put forward. 
There is a strong feeling that these issues hurt the effectiveness of the residence program in terms of educational outcomes.

Moreover, ongoing specialization of hospitals towards provision of specific care services implies that internships required for residents' qualification can no longer be offered by a single hospital. This emphasizes the need for regional coordination of residents' activities among district hospitals and academic hospitals. The departments of these hospitals that are involved in an educational program have to co-ordinate their involvement. However, it is not just the planning and scheduling of individual residents that needs to be resolved, but also the interest of these departments for their role in the educational programs of the residents. Moreover, the implementation of the concept of personalized learning, i.e., tailoring the program to individual resident's qualities and progress, work-life balance, and part-time employment, strongly increases scheduling detail.

One of the more recent factors that impacts planning and scheduling strongly is the measurement of learning outcomes and its impact on the resource planning in hospitals. We observe a tendency to measure outcomes of educational programme for residents, e.g., in the US, Australia, The Netherlands (Englander \& Carraccio, 2014), using Entrustable Professional Activities (EPA’s). EPA’s reflect specific competencies, skills, knowledge, and attitudes required for a professional task with a recognizable output. They focus on actual physician tasks and can be assessed by experts. The trust levels indicate whether trainees are ready for the next milestone, such as unsupervised practice, based on assessment of performance of those activities. Typically, five or more milestones (i.e., levels) of these tasks are distinguished and minimum performance levels for trainees may differ. EPA's introduce additional planning complexity due to the inherent uncertainty on when new levels are being acquired, which has an impact on the availability requirements of residents and their supervisors. At the other hand, planning and scheduling may become easier as well due to the possibilities that the systematic monitoring of skills available among the physicians and residents offers. Nevertheless, the change towards EPA's has consequences for planning and scheduling and raises several issues. This study explores these issues by using several data sources and qualitative research methods that will be discussed in the next section.

\section{Data sources and research methodology}

Each educational programme that uses or plans to use EPA's in programme delivery and assessment needs to describe the programme outcomes and assessment process. We selected a Dutch Residency programme on radiology that has described their programme in terms of EPA's. Moreover, the programme director has been interviewed in order to better understand the choices made when redesigning the programme. 
Next, interviews with several residents have been issued. The main question during these interviews related to the planning and scheduling issues when a change towards EPA's and more personalized learning would be facilitated. This resulted in an inventory of issues, requests, and bottlenecks that they encountered.

Finally, we investigated amongst schedulers how they perceived the problems (current and future). Note that the scheduler could be one of the residents, a department secretary, or even a supervisor, depending on the size, organization, and complexity of the department and the educational programme.

For developing a new framework for planning and scheduling, we used design methodology.

\section{Results}

\subsection{Entrustable Professional Activities in the programme of Radiology}

The analysis of the educational programme of Radiology revealed that it entails 120 EPA's, divided amongst eight themes that are all necessary for an all-round radiologist. For each theme a minimum length of the theme-internship is determined, as well as a list that specifies how frequently specific professional activities on average have to be performed, reported and reflected upon as part of the educational programme. Moreover, each radiologist may select up to two themes for a differentiation. Within the differentiation, a higher level of some EPA's need to be achieved, accompanied with longer internships within these themes. This is made explicit in the programme description. Once a resident starts in a new theme at EPA level 1 (not allowed to perform, not even under supervision), a pre-test is taken. When passed, the resident is allowed to accompany an trained educator and observe how the activities are done. After achieving the second EPA level (allowed to perform, but under direct supervision) the resident is allowed to accomplish the task under direct supervision. The supervisor will take the lead in providing feedback at these two EPA levels and is present during the whole task. The third EPA level (allowed to perform the set of activities with indirect supervision) is the next trust level a resident can achieve. From this EPA level onwards, the initiative to receive feedback is with the resident itself. The supervisor does not have to be at the same location, but has to be available for feedback. For highly complex and less frequent tasks, this level may be the highest to achieve for a resident that will not specialize in this theme. However, for frequent and less complex tasks, EPA level 4 is the standard for all residents: allowed to perform unsupervised. Residents that specialize in a theme have to achieve even EPA level 5 for these frequent tasks: allowed to supervise.

\subsection{Residents perception of scheduling issues}

The residents indicate that due to their personal circumstances (i.e. family planning), most of them prefer to work part time (80\%), which in practice means that their number of working 
and studying hours per week are similar to full time jobs outside the clinic, as 38 hours per week is the normal number of working hours in The Netherlands for full time employees. Previously (i.e. a decade ago), the majority of the residents worked full time. This has consequences for planning of internships and the length of an internship, as it affects the build up of expertise and frequency of practicing some acitivities. However, schedulers do not always take this into account. Moreover, they are sometimes involved in all kinds of unscheduled activities, such as making their own rosters, preparing meetings, administration, and organizing activities, which would normally have been accomplished by support staff from the department or hospital. Due to budget cuts, this kind of tasks have to be done by the residents themselves, which takes a lot of time.

Finally, the main stressor for residents is the uncertainty with respect to their programme as well as regular care services they have to provide. To proceed to another level of an EPA, a minimum of two supervisors need to sign off and approve that the resident is entrusted to a higher level of autonomy. This is not just based on number of activities that have been performed and reflected upon, but involves an overall assessment. This increases uncertainty for the resident. With respect to the regular patient care services, it is mainly the uncertainty of additional care services they have to fulfill, due to unavailability of other staff members. This uncertainty causes a lot of stress due to the organization of private life (child caretaking for example).

\subsection{Schedulers and their perceptions on the residents' scheduling problem}

The schedulers report that the problem consists of several layers. They receive inputs from the head of the department on the internships that have been planned for the next months, as well as the available capacity of the regular staff of the department. Next they determine a schedule with a horizon of three months that details the who, when and where of the planned availability of the residents, supervisory staff, and other staff in this department of the hospital. Based on the three-month plan, detailed short term plans are extracted in which attention is given to both the educational activities and the total care delivery, also in case no direct supervision is available (weekends, night shifts, et cetera).

The main problem the schedulers face is the amount of rework in the scheduling process. They receive some information too late, changes are required while they had not been informed, et cetera. The schedules are rather detailed but not accurate. The second problem is the lack of anticipation capability on capacity shortages. As schedulers, they are not able to fill these capacity shortages, so they deliver incomplete schedules. Finally, changes to another EPA level causes major changes in the capacity and flexibility of staff in the department, but are difficult to include in the schedules due to the aggregation level of these schedules. For the lower EPA levels, tow scheduling (trainee and trainer) has to be applied, 
while at higher EPA levels, single skilled operator scheduling would be possible (Hadjaissa, Ameur, Ait cheikh, \& Essounbouli, 2016).

\section{Framework, conclusion and future research}

Based on the findings of the various stakeholders, we have developed a new framework for planning and scheduling of residency programs. The framework depicts some major changes in the way plans and (more detailed) schedules are to be produced, what actors will have to take the lead in what stage of plan development, and what will be the main decisions to take. It is based on lean planning principles as described in literature (Riezebos, 2018). The main changes we propose are:

1. Improved regional coordination in order to enable personalization and speed-up of residency programs.

2. Program coordinator in the lead: better planning of EPA's per resident, enabling residents to achieve the intended learning outcomes and competencies per EPA level.

3. Intelligent support of planning and scheduling: use algorithms to reduce changes in the timing aspect of planning decisions (when to provide service or be involved in educational activities), while allowing for changes in the what and where of these decisions (what activities in which department). Examples of intelligent support can be found in e.g., (De Bruecker, Van Den Bergh, Beliën, \& Demeulemeester, 2015; Erhard, Schoenfelder, Fügener, \& Brunner, 2018; Stolletz \& Brunner, 2012; Topaloglu, 2006; Van Den Bergh, Beliën, De Bruecker, Demeulemeester, \& De Boeck, 2013; White, Nano, Nguyen-Ngoc, \& White, 2007).

The framework that we propose is depicted in Figure 1.

At the left hand side of the framework, we have allocated the central role of program coordinator and regional coordination between hospitals where the internships can be performed. Not all themes can be performed at every hospital in the region. And for complex care, the expertise and possibility to develop skills might only be available in the academic hospital. This requires coordination in order to facilitate the individual preferences for learning of the residents as well as the capacity of educators in the various hospitals. The result will be a grouping of residents per location and per theme.

The middle part of the framework depicts the planning and scheduling activities within departments where one or more themes can be trained. Here, EPA planning will be the basis for developing capacity plans and schedules. Task allocation (i.e., what EPA to accomplish when and by whom) and a detailed schedule of the timing a resident has to provide care services during the internship and regular care of the department (night and weekend shifts) 
has to be accomplished and produced. Based on these plans, a more detailed analysis of required capacity of the regular staff can be made.

At the right hand side of the framework, the residents themselves will have to decide who will take what care services at the specified time slots. They will take their own progress in terms of EPA's into account when selecting the location and activities in the department for the specific time slot. The main uncertainty with respect to the timing of their work hours has been addressed at an earlier moment, but the decision what activities to perform can be postponed. Next, their decisions can be used as input for the physicians that co-operate with the residents in the patient care operations, as they might be able to support the residents in making the most of their regular care services in terms of building expertise, challenging their learning process, and finding evidence from new cases.

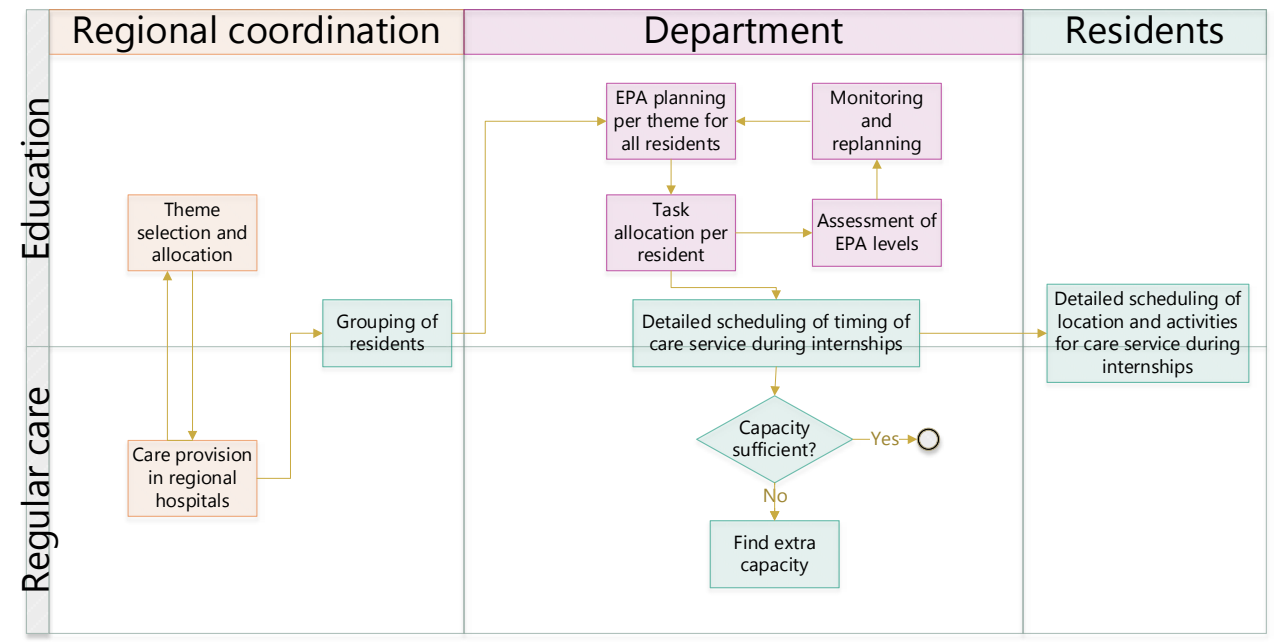

Figure 1. Framework for planning and scheduling of residency programs and activities

Based on this new framework, people can be trained and algorithms can be developed to support the decision making at the various stages of plan development.

We conclude that the problem of planning and scheduling of resident activities in programs that have implemented EPA's needs a change in the way hospitals plan the activities of residents. Within a theme, some 8-10 EPA's might be formulated and each EPA distinguishes five levels at which the activity is allowed to be performed by the resident. Each level corresponds with requirements on the availability of supervisors that are able (i.e., allowed) to provide feedback and assess. Not every level can be achieved at every hospital in the region, so the resulting planning problem is complex and needs to be supported by a systematic process, including training and algorithmic support. 
The main discussion point is whether hospitals realise that a fundamental change in the assessment procedure towards EPA's will have a major impact on the way they plan and schedule their educational programmes and their care service operations, the capacity problems that will arise, and the responsibility referents will take for their own education.

\section{Acknowledgements}

We would like to express our thanks for the insights and information provided by residents, program coordinators, and schedulers at the University Medical Center Groningen, The Netherlands. We thank Babette Huisman for her role in interviewing several of these people.

\section{References}

Cohn, A., Root, S., Kymissis, C., Esses, J., \& Westmoreland, N. (2009). Scheduling medical residents at Boston University School of Medicine. Interfaces, 39(3), 186-195.

De Bruecker, P., Van Den Bergh, J., Beliën, J., \& Demeulemeester, E. (2015). Workforce planning incorporating skills: State of the art. European Journal of Operational Research, 243(1), 1-16.

Englander, R., \& Carraccio, C. (2014). From theory to practice: Making entrustable professional activities come to life in the context of milestones. Academic Medicine, 89(10), 1321-1323.

Erhard, M., Schoenfelder, J., Fügener, A., \& Brunner, J. O. (2018). State of the art in physician scheduling. European Journal of Operational Research, 265(1), 1-18.

Ernst, A. T., Jiang, H., Krishnamoorthy, M., \& Sier, D. (2004). Staff scheduling and rostering: A review of applications, methods and models. European Journal of Operational Research, 153(1), 3-27.

Güler, M. G., Idin, K., \& Yilmaz Güler, E. (2013). A goal programming model for scheduling residents in an anesthesia and reanimation department. Expert Systems w. Applications, 40(6), 2117-2126.

Hadjaissa, B., Ameur, K., Ait cheikh, S. M., \& Essounbouli, N. (2016). Bi-objective optimization of maintenance scheduling for power systems. International Journal of Advanced Manufacturing Technology, 85(5-8), 1361-1372.

Hauer, K. E., Soni, K., Cornett, P., Kohlwes, J., Hollander, H., Ranji, S. R., Sullivan, P. S. O. (2013). Developing Entrustable Professional Activities as the Basis for Assessment of Competence in an Internal Medicine Residency. Journal of General Internal Medicine, 28, 1110-1114.

Riezebos, J. (2018). Lean Planning and Control: Principles and Practice. In G. Suer \& M. Gen (Eds.), Cellular Manufacturing Systems, recent developments, analysis and case studies (pp. 517-531). New York: Nova Science Publishers.

Stolletz, R., \& Brunner, J. O. (2012). Fair optimization of fortnightly physician schedules with flexible shifts. European Journal of Operational Research, 219(3), 622-629. 
ten Cate, O. (2013). Nuts and Bolts of Entrustable Professional Activities. Journal of Graduate Medical Education, 5(1), 157-158. 1

Topaloglu, S. (2006). A multi-objective programming model for scheduling emergency medicine residents. Computers and Industrial Engineering, 51(3), 375-388.

Van Den Bergh, J., Beliën, J., De Bruecker, P., Demeulemeester, E., \& De Boeck, L. (2013). Personnel scheduling: A literature review. European Journal of Operational Research, 226(3), 367-385.

White, C. A., Nano, E., Nguyen-Ngoc, D.-H., \& White, G. M. (2007). An Evaluation of Certain Heuristic Optimization Algorithms in Scheduling Medical Doctors and Medical Students. Practice and Theory of Automated Timetabling VI, 105-115. 